\title{
CONTRIBUTION TO SAFETY OF NAVIGATION BY INTRODUCTION OF NEW TECHNOLOGIES IN FAIRWAY
}

\author{
Pero Vidan, Mirko Čorić, Anita Gudelj, Srđan Vukša* \\ Faculty of Maritime Studies, University of Split, Split, Croatia
}

Navigation in archipelagos and shallow waters is considered dangerous. Although islands, reefs, and shallows can be properly marked by lights and other navigation aids, skippers and other non-professional navigators usually do not have sufficient experience for safe navigation. Available navigation aids such as radars, Electronic Charts Display and Information System (ECDIS), and alike are usually expensive for use on small boats and pleasure crafts. In this paper, the authors propose a new system of identifying navigation marks that would significantly reduce the possibility of maritime accidents and errors due to a wrong interpretation or invisibility of the mark. The key part of the new system proposed refers to an innovative method that identifies, delivers, and displays in a user-friendly manner those navigation marks that are relevant to skippers and other non-professional navigators, depending on their location during navigation. The proposed system would be cheaper than the professional SOLAS-approved system, and available on the web.

Key words: safety, fairway, navigation, navigational marks

\section{INTRODUCTION}

The existing marking system is tailored to professional seafarers and the Convention ships, and has been established by the International Association of Marine Aids to Navigation and Lighthouse Authorities regulations [1]. Professional seafarers are subject to medical examinations and certifications required for the renewal of seamen's books and certificates. These have a uniform health check-up system and experience in recognizing marks. Boat masters and skippers do not have the necessary navigation experience, training and licensing procedures are less demanding, and they do not fall under the medical examination system [2]. During the high season, archipelago seas are very popular among tourists. Therefore, in the waters mentioned, there is the largest number of smaller (non-Convention) vessels and the largest number of maritime accidents.

All vessels of more than 300 Gross tonnage (GT) and passenger vessels irrespective of their size are required to be equipped with at least one radar. Electronic Chart Display and Information System (ECDIS) is required for all passenger vessels of more than $500 \mathrm{GT}$ although the administration can lay down stricter requirements for its own fleet than those of the International Convention for the Safety of Life at Sea (SOLAS) [3, 4]. However, the majority of smaller vessels used as pleasure crafts are not legally required to carry any of the above mentioned devices $[5,6,7]$.

The skippers of smaller leisure crafts usually have insufficient experience. They sometimes sail at night and in conditions of reduced visibility. In such conditions, the probability of navigation errors due to a wrong interpretation of navigation marks is significant. The prices of modern and SOLAS-approved navigational devices might be high for small boats. Therefore, different producers are trying to replace such devices with new facilities, not SOLAS-approved and with different standards. Such systems are usually based on GPS positioning of ships and marking of objects on digital charts (plotters, etc.). There are no information about navigation marks, or advice, etc. with such devices. In this article, the authors propose a software for easy recognition of navigation marks on the spot and advice for navigation using GPS, and tablets or mobile phones. This kind of software should make navigation easier and reduce human error, especially for non-professional navigator during high season period.

\section{BASIC CONCEPT OF THE METHOD}

For a navigation area, relevant points are determined in advance, i.e. the software stores the Global Positioning System (GPS) coordinates of relevant points. The number of relevant points, their distribution and density can vary, and the navigation area can be filled by relevant points on the basis of different criteria (e.g. even addition, addition with regard to the density of maritime traffic in the area, number of navigation marks in the area, indebtedness of the coast, geography, etc.). For each relevant point in a navigation area the visibility of navigation marks in the vicinity is determined, i.e. navigation marks visible for a relevant point are explicitly programmed. A vessel under way performs in short time intervals the selection in its program of which of the relevant points is closest to it, and on the navigation display only those navigation marks visible for the relevant point can be seen. The illustration of the fundamental idea of the method is shown in the Figures 1, 2 and 3.

The proposed method for solving the above mentioned problem represents a combination of satellite and e-nav- 


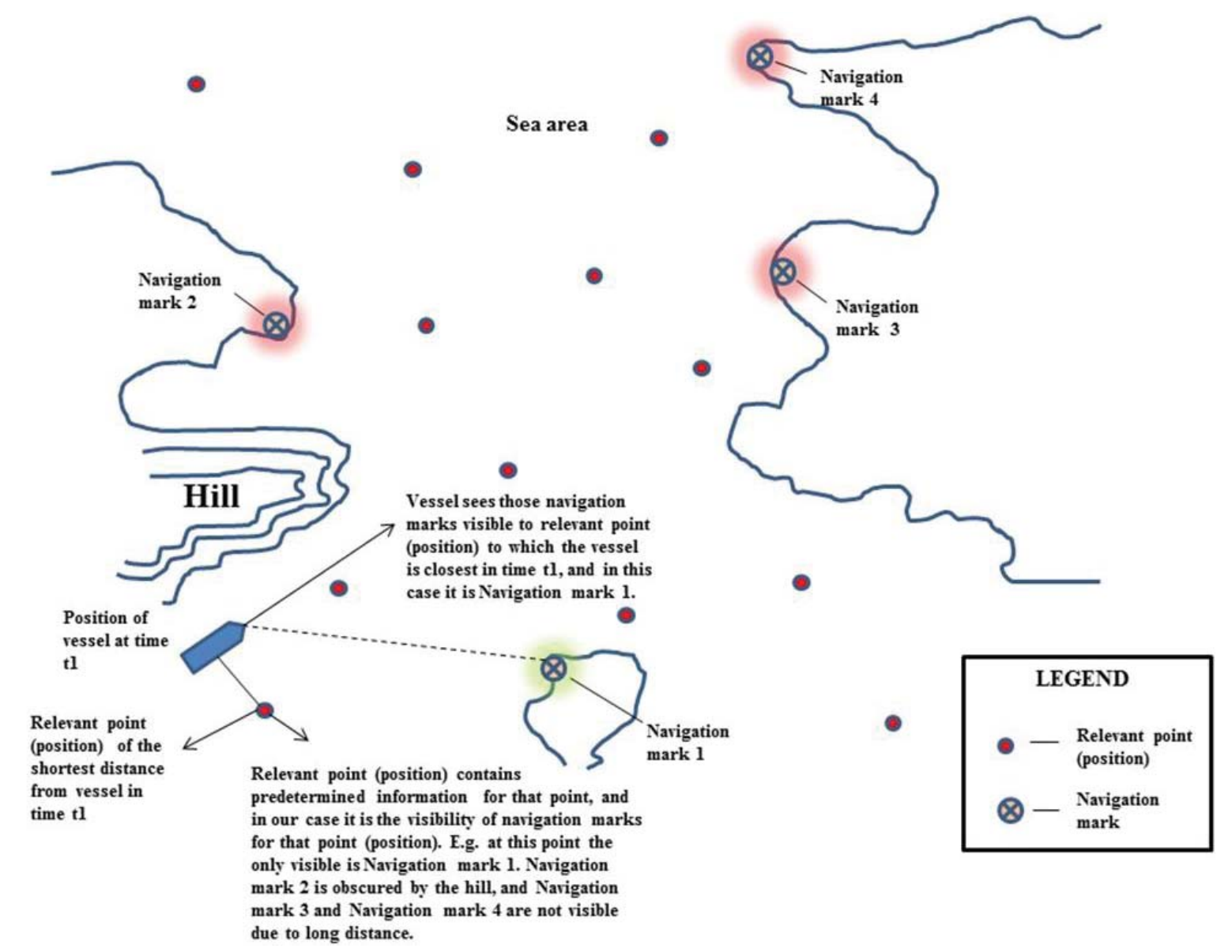

Figure 1: Vessel at time t1 (Source: Authors)

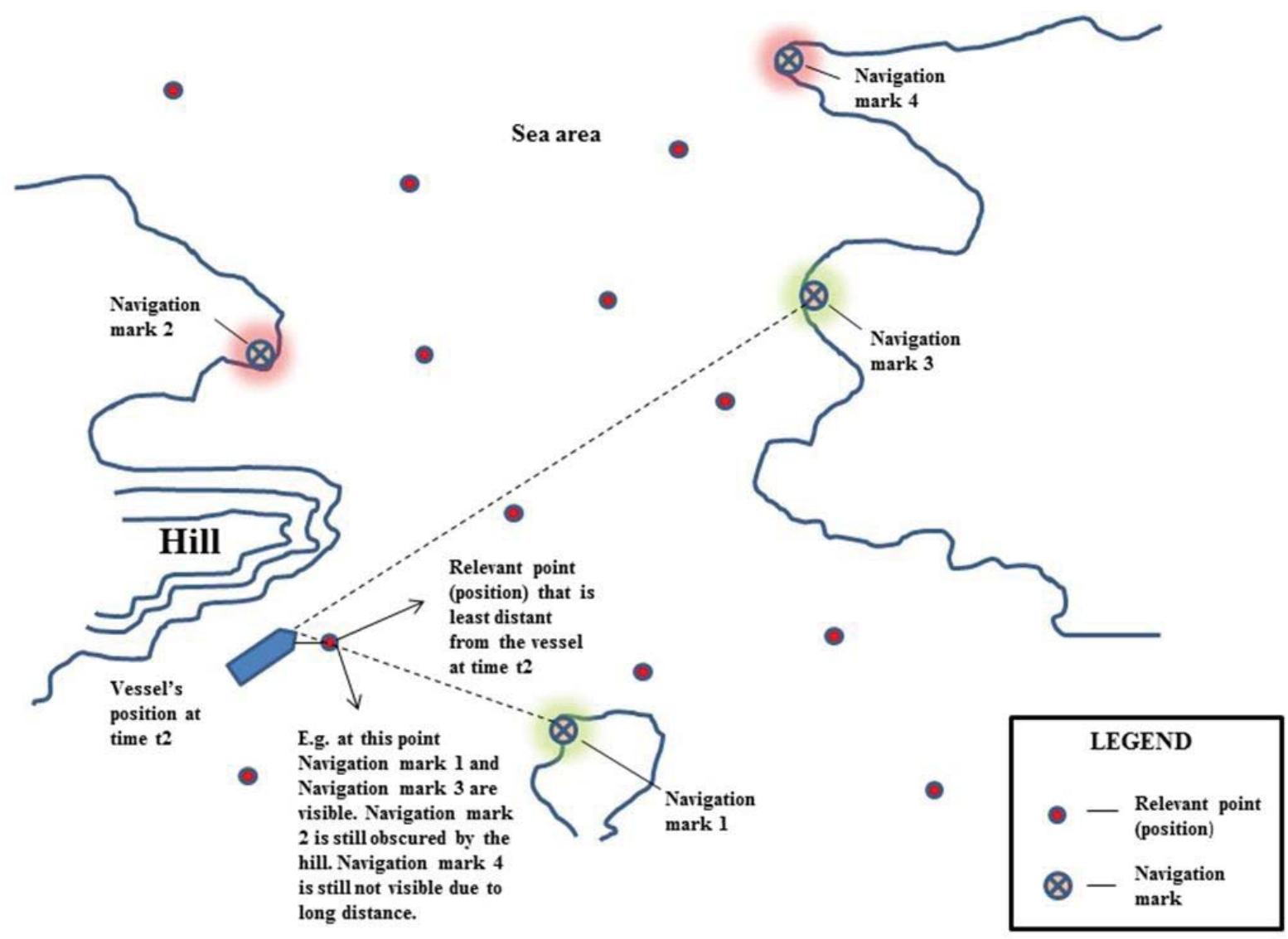

Figure 2: Vessel at time t2 (Source: Authors) 


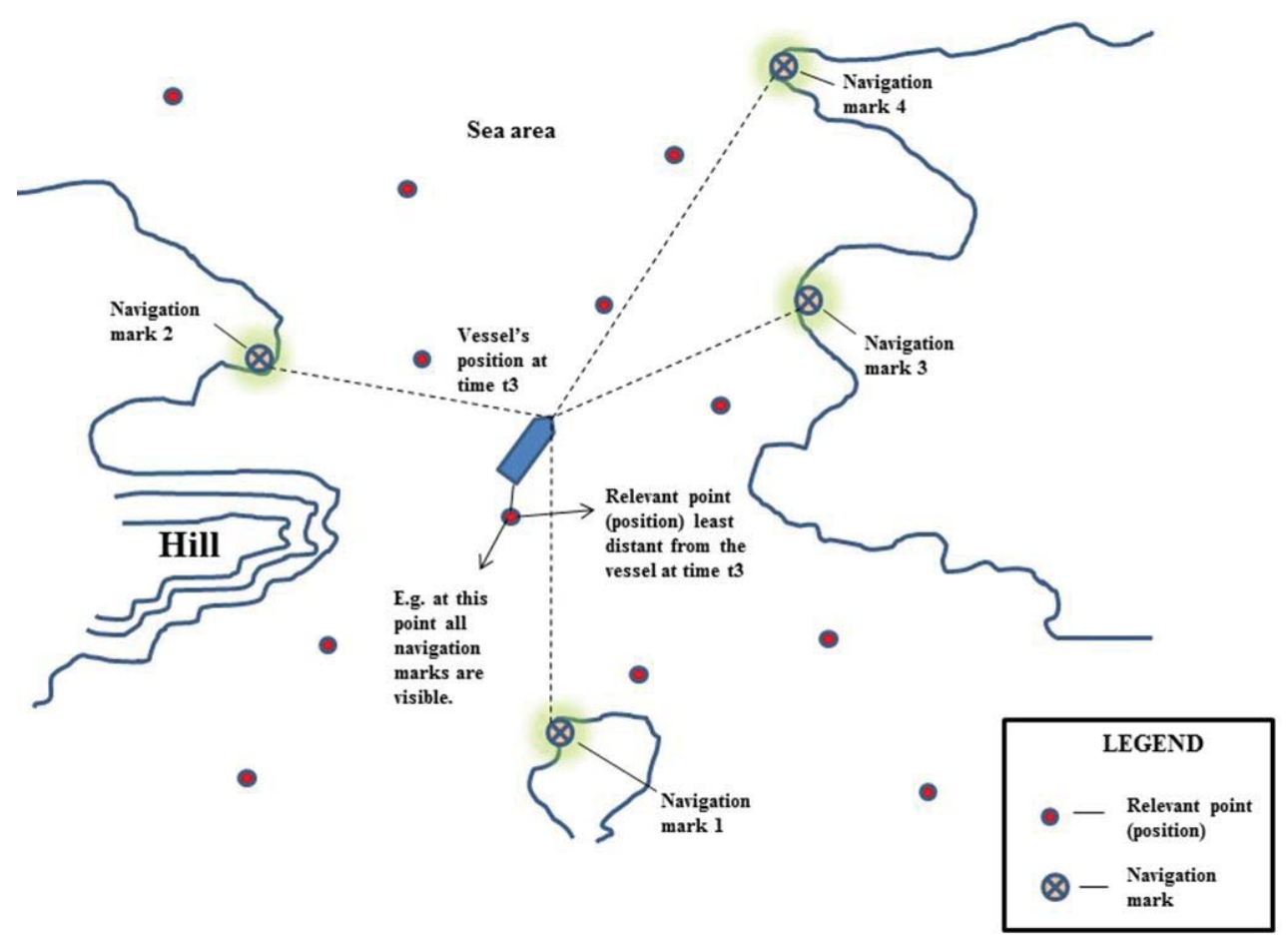

Figure 3: Vessel at time t3 (Source: Authors)

igation. Providing that the area has internet access, a reasonably priced and practical solution is proposed in the form of web service to enable the above mentioned method, and user applications (applications used by seafarers, e.g. skippers on boats) to communicate with the web service (Figure 4).

The approach proposed allows user applications that would be used by seafarers to communicate with the web service to be implemented in different ways. It can be an application started on the mobile phone, tablet, computer or any other device (connected to the internet)

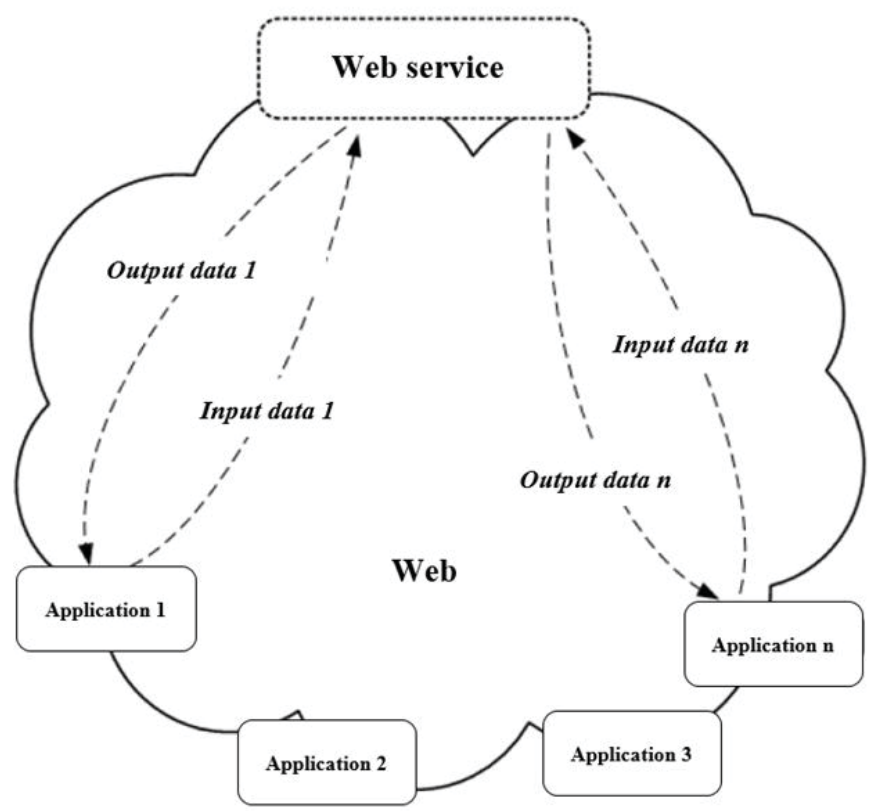

Figure 4: The concept of web service and ship's applications using it (Source: Authors) that communicates with the web service while the vessel is under way. Thus, the shipboard application initiates the session, i.e. communication with the web service, and the session lasts as long as the vessel is under way. It is proposed that the user application to be used by seafarers on board has a graphical interface with the division into three parts to indicate navigation marks, their distances, and bearings of navigation marks relative to the vessel's course as proposed on Figure 12 [8]. Also, it is proposed that the method is realised/implemented as a web service, and available on the internet together with a database of relevant points. This would mean that the user (shipboard) application would not perform any calculations, but it would have the role of a graphical interface for the display of data related to navigation marks, provided by the web service while under way, and to which the application would communicate in such a way that during the session (navigation) it would transmit to the web service the vessel's GPS coordinates, while the web service would provide back to the application the data related to the visibility of navigation marks for that particular GPS coordinate.

From the fundamental idea of the method and the problem posed (Figure 1, Figure 2 and Figure 3), it is clear that the distances between GPS coordinates of the vessel and GPS coordinates of the surrounding points (GPS coordinates of the relevant points and GPS coordinates of navigation marks) are small enough to enable the curvature of the Earth to be entirely neglected in the calculation.

Therefore, all the laws based on spherical trigonometry (the Haversine formula, Great Circle distance, etc.) can be neglected $[9,10]$, and the problem posed can be observed in 2- dimensional Cartesian coordinate system 
[11]. Also, one easily comes to a conclusion that the accuracy of the method applied will be proportional to the number of relevant points.

\section{METHOD ANALYSIS AND POSSIBLE ISSUES}

The key part of this method is finding the relevant point, i.e. its GPS coordinates closest to the GPS coordinates of the ship's position. Thus, it is necessary to compare the GPS coordinates of the vessel's position with a potentially large number of the GPS coordinates of the relevant points and in doing so, find the one that is closest to the GPS coordinates of the vessel's position. This is done in the way that the Euclidean distance $[12,13]$ between the point marking the vessel's position and the relevant points is calculated. The Euclidean distance is generally described as follows:

The Euclidean distance between the points and is the length of the segment of the line connecting them $(\overline{p q})$. In the Cartesian coordinate system,

$p=\left(p_{1}, p_{2}, \ldots, p_{n}\right)$

and

$q=\left(q_{1}, q_{2}, \ldots, q_{n}\right)$

are two points of the Euclidean $n$ - space, then the distance $(d)$ from $p$ to $q$, or from $q$ to $p$ is default by the Pythagorean Theorem:

$d(p, q)=d(q, p)=$

$=\sqrt{\left(q_{1}-p_{1}\right)^{2}+\left(q_{2}-p_{2}\right)^{2}+\ldots+\left(q_{n}-p_{n}\right)^{2}}=$

$=\sqrt{\sum_{i=1}^{n}\left(q_{i}-p_{i}\right)^{2}}$

In our case, the Euclidean distance should be calculated for two dimensions only. For $p=\left(p_{1}, p_{2}\right)$ and $q=\left(q_{1}, q_{2}\right)$ the two-dimensional Euclidean distance is:

$$
d(p, q)=\sqrt{\left(q_{1}-p_{1}\right)^{2}+\left(q_{2}-p_{2}\right)^{2}}
$$

which is equivalent to the Pythagorean Theorem.

This part of the method that calculates the distances is computationally the most demanding due to root calculation and a potentially large number of relevant points [13].

The analysis mentioned above suggests that root calculation during search for the minimum distance between the point indicating the vessel's position and the relevant points is to be avoided due to high processor computational complexity. In our case, where we actually only compare the Euclidean distances and search for the shortest among them (values of lengths are not important), for optimization reasons it is sufficient enough to calculate and compare the squares of the Euclidean distances:

$$
\begin{aligned}
& d^{2}(p, q)=d^{2}(q, p)=\left(q_{1}-p_{1}\right)^{2}+\left(q_{2}-p_{2}\right)^{2}+ \\
& +\ldots+\left(q_{i}-p_{i}\right)+\ldots+\left(q_{n}-p_{n}\right)^{2} \\
& \text { For } n \text { - dimensional space, i.e.: }
\end{aligned}
$$

$$
d^{2}(p, q)=d^{2}(q, p)=\left(q_{1}-p_{1}\right)^{2}+\left(q_{2}-p_{2}\right)^{2}
$$

For 2-dimensional space in our case.

The other problem mentioned above is a potentially large number of relevant points. This problem can be solved by the division of the coastal area considered, i.e. of the nautical chart, into smaller areas, i.e. sectors. In this way, a potentially large area with a potentially large number of relevant points can be divided into a number of smaller areas with a smaller number of relative relevant points. In that case, the minimum distance would be calculated between the point marking the vessel's position and the relevant points of the sector in which the vessel is currently found, and all the relevant points of the other sectors would be neglected.

The following analysis gives an insight into the dependence of the search time for the minimum distance on the number of relevant points among which the minimum distance is to be found (Figure 5). It also shows what happens the search time of the minimum distance when the number of sectors increases in case the above mentioned criterion is applied which proposes the division of the area observed into approximately equal rectangular areas (sectors) with an approximately equal number of relevant points across a rectangular area (sector) (Figure 6). For the following analysis, it is not essential that the rectangular areas (sectors) be of equal size, but that each sector has an approximately equal number of relevant points. Equal sizes of sectors are recommended for simplicity sake.

From the Figure 5 and the data it is clear that with an increase in the number of relevant points, the minimum distance calculation time increases slightly exponentially. So, in case 8,000 relevant points are used for the area observed, finding a relevant point closest to the point

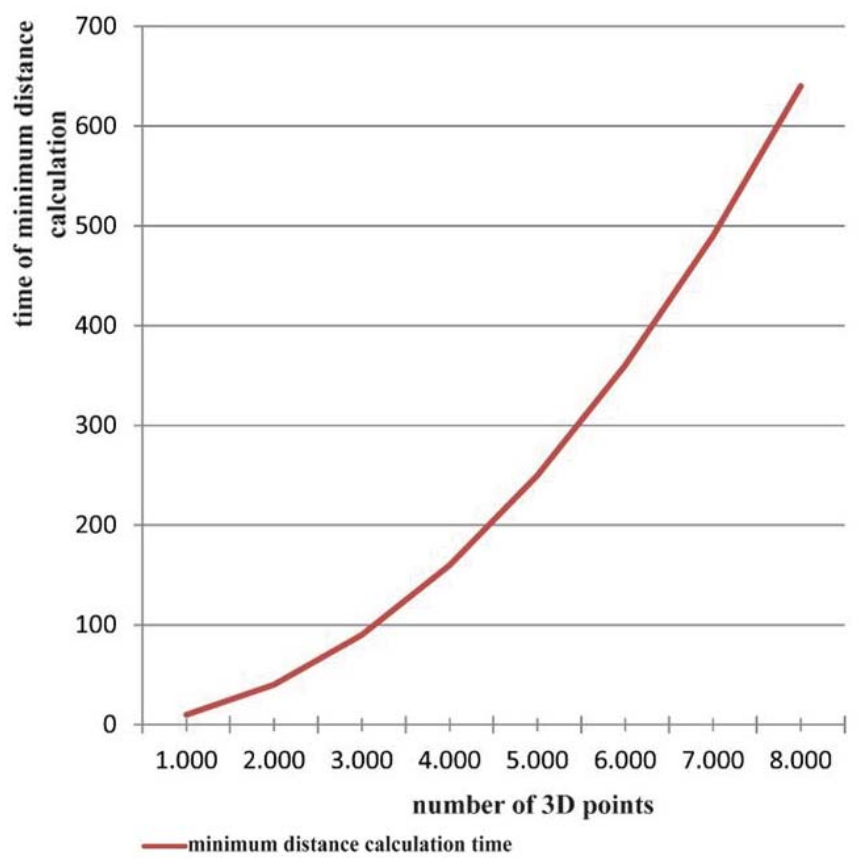

Figure 5: Dependence of minimum distance calculation time on the number of 3D points (Source: Authors) 


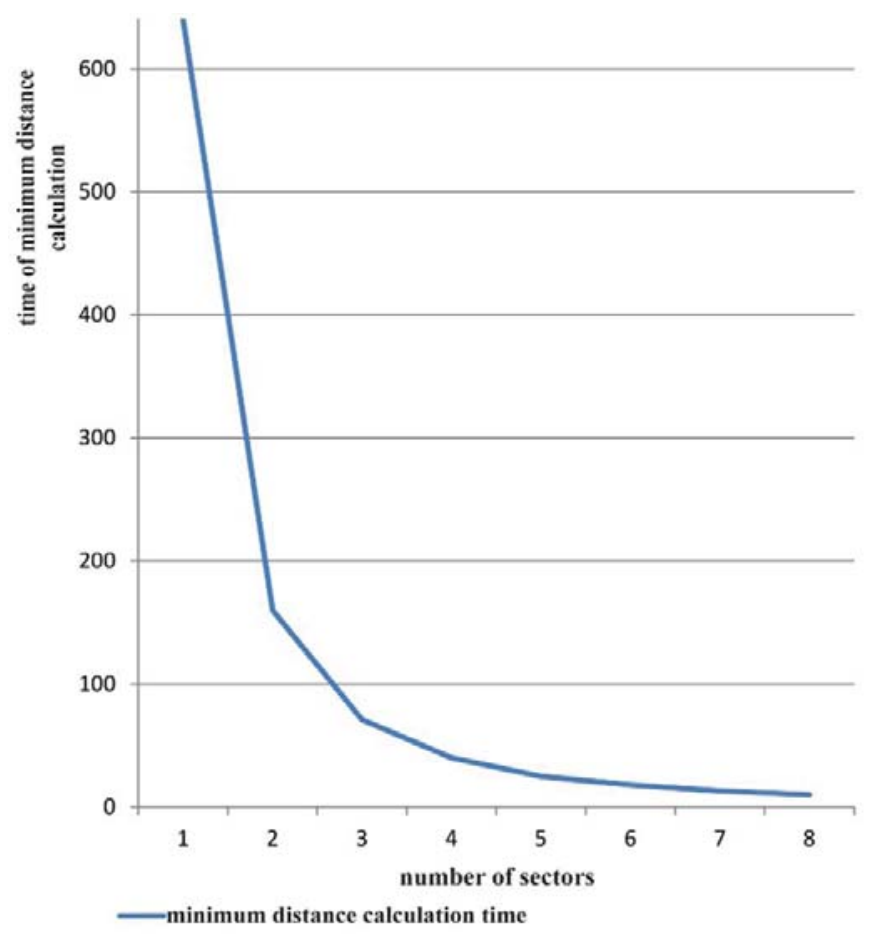

Figure 6: Dependence of minimum distance calculation time on the number of sectors (Source: Authors)

of the vessel's position will take 640 seconds. These minimum distance calculation times can be drastically shortened if the above mentioned division of the area observed into sectors is used.

Figure 6 shows the dependence of minimum distance calculation time on the number of sectors with the use of 8,000 points and approximately even distribution of points across sectors.

With an increase in the number of sectors, the minimum distance calculation time is logistically reduced. It is obvious that the maximum reduction of the minimum distance calculation time (the steepest fall of the curve) is achieved by introducing only a few sectors. With a further increase in the number of sectors the calculation time is further reduced, but less intensively. From the example it is clear that by entering only 5 sectors the minimum distance calculation time was reduced by approximately $96 \%$.

The next question posed is the type of coordinate system to be used for the display of GPS coordinates of the points (the point of vessel's position, relevant points and the points in which there are navigation marks). As our problem refers to 2D Cartesian coordinate system, it is logical to use the projection coordinate system, and the method has been designed to use Universal Transverse Mercator (UTM) coordinate system [14, 15]. UTM coordinate system has been chosen because it is simple and entirely meets the requirements of our method so that the observed geographical area is reduced to a common 2D Cartesian coordinate system with two axes. UTM coordinate system uses meters as the unit of measurement (i.e. the need for units of measurement such as degrees, minutes, seconds is eliminated). The coordinates of a point on the Earth is represented by a segment on the horizontal x-axis ("easting") and segment on the vertical $y$-axis ("northing"). In the calculation, the need for spherical trigonometry is eliminated, i.e. simple mathematics and trigonometry are used just like in the $2 \mathrm{D}$ Cartesian coordinate system. UTM coordinate system does not present negative values, i.e. GPS coordinates of points are always represented as positive values.

UTM coordinate system divides the Earth's surface into 60 rectangular UTM zones, and each zone is a large rectangle whose two longer sides extend across both hemispheres (ellipsoid meridians are ignored), intersecting the Equator at right angles (Figure 7).

The entire large rectangle shown in Figure 7 represents one UTM zone. Provided we are in the Northern hemisphere, then the origin can be taken to be the point in which the Equator and the upper left outer limit of the large rectangle meet. In other words, the Equator is x-axis, the upper left outer limit of the rectangle y-axis. Provided we are in the Southern hemisphere, then the origin can be considered to be the point in which the lower outer limit of the large rectangle joins the lower left outer boundary of the rectangle. The actual coordinates of a point from the zone observed will always be within the red ellipsoid from the Figure 7 . Therefore, redundancy occurs on the segments on both axes, and this means that the actual point from the zone observed will never be found at the very boundaries of the zone. It is essential to emphasise that each UTM zone is a particular coordinate system and there is no mathematical relation

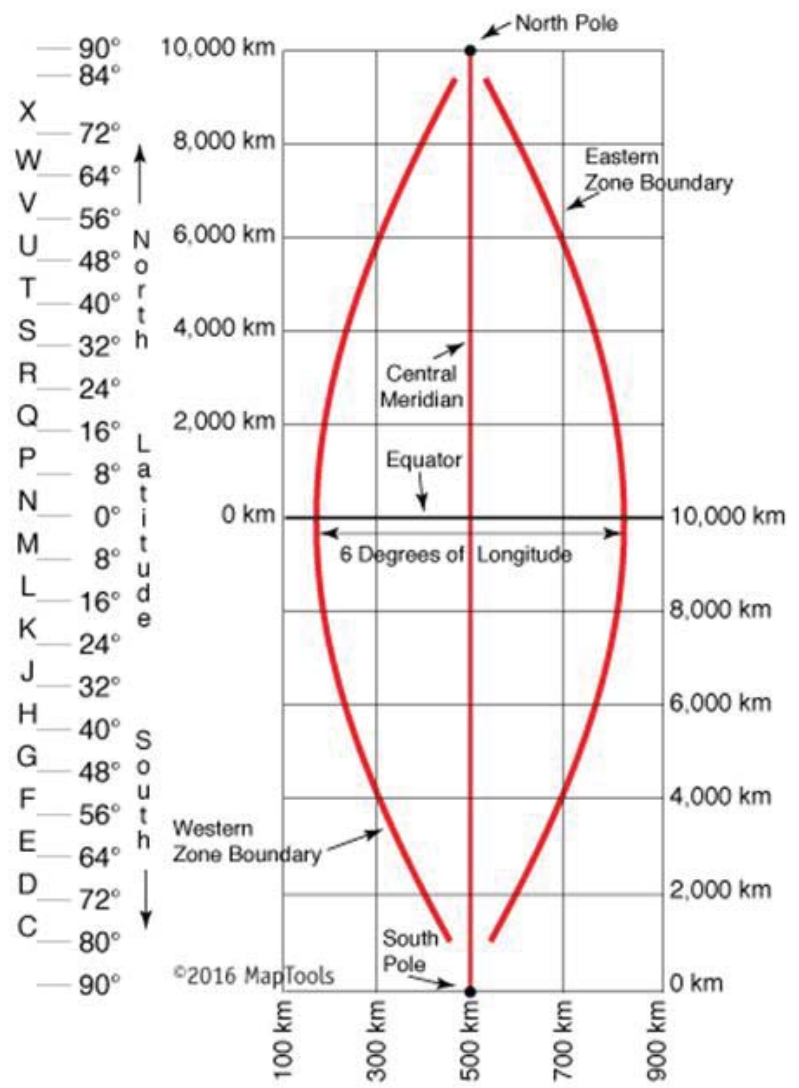

Figure 7: Example of UTM zone [16] 
with the other coordinate systems, described in detail by Department of Geography (2018) [15] and Map Tools (2019) [16].

From the theoretical point of view, it is possible that the area observed to which we intend to apply the method extends across two or even more UTM zones. In that case, we are able to assemble our rectangular areas (sectors) like "puzzles", so that they accurately match the boundaries of UTM zones, not intersecting them. In this way there is no possibility during the minimum distance calculation between the relevant points within a rectangular area (sector) for a relevant point to be found in one UTM zone, and another relevant point in a different UTM zone. For example, let the small rectangles within UTM zone (of the large rectangle) from Figure 7 represent our rectangular areas (sectors). Then, Figure 8 clearly illustrates the concept mentioned by which the possibility will be eliminated that the rectangular area (sector) intersects UTM zone.

The next problem encountered is the case in which the vessel is found close to the edge of the sector in which it plies. Namely, in such a situation there is the possibility that at a certain point during the calculation of the relevant point which is closest to the vessel at that instant, the vessel can actually be closer to a relevant point from the adjacent sector. In that case, due to the way in which the method described in this paper operates an error will occur because the closest relevant point of the sector in which the vessel is found will be considered the closest relevant point, and not the actual closest relevant point from the adjacent sector. The situation is shown in Figure 9.

A satisfactory solution of this problem can be found so that a larger number of relevant points are located closer to the boundaries of the sector. In that case the proba-

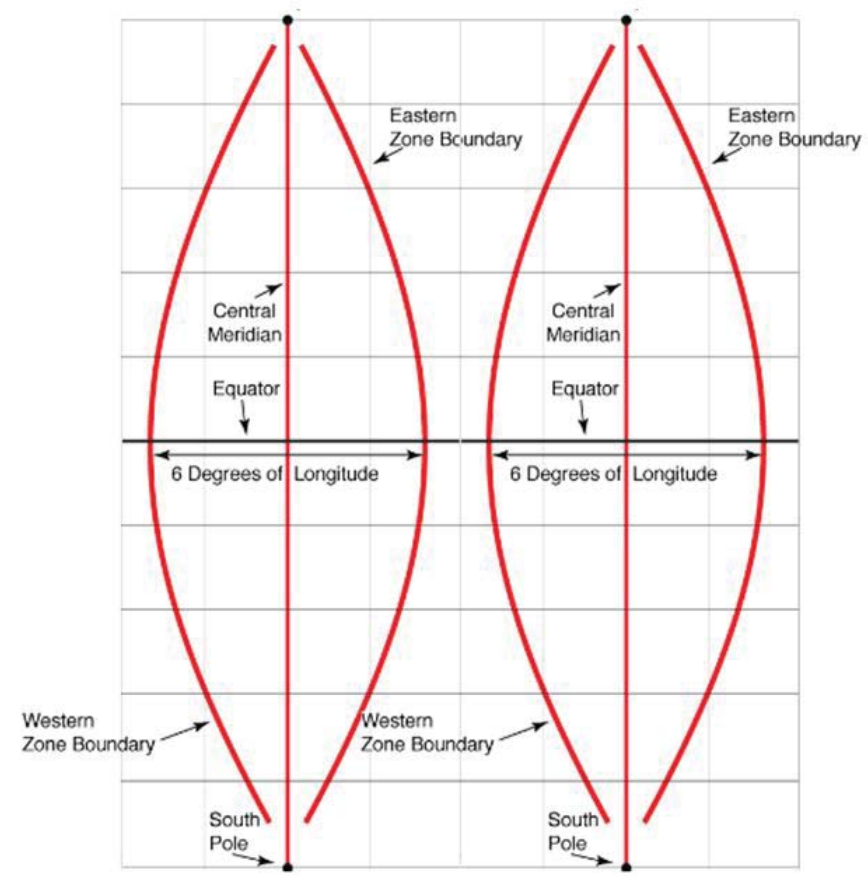

Figure 8: Illustration of a possible sector distribution to avoid overlapping of UTM zones [16]

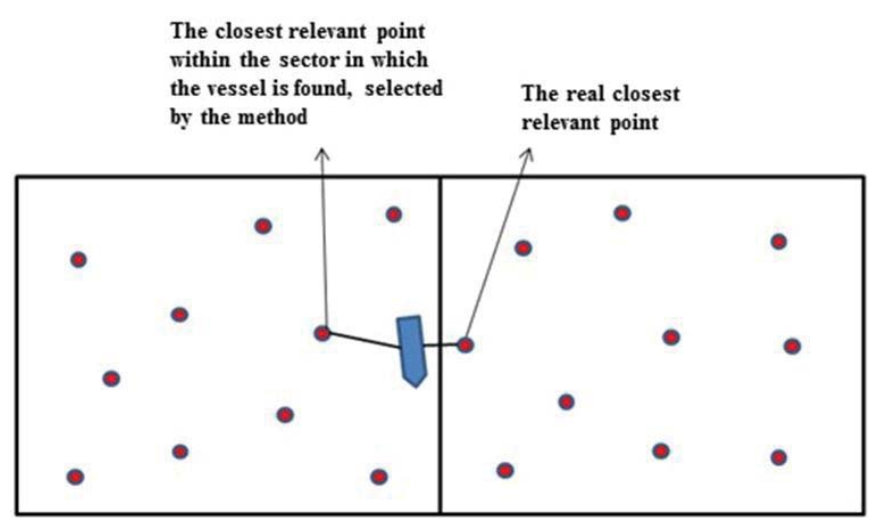

Figure 9: Potential situation in which an error occurs in the calculation of the closest relevant point (Source: Authors)

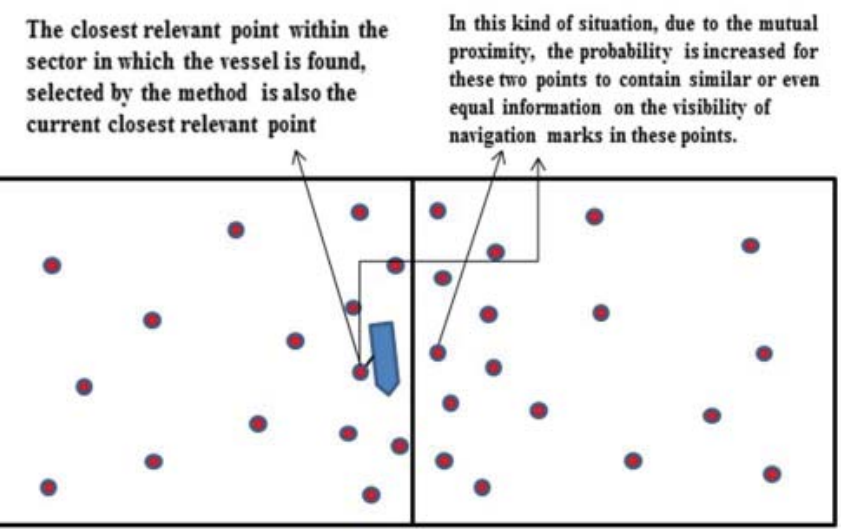

Figure 10: Proposed concept of reducing the probability of error in calculation of the closest relevant point (Source: Authors)

bility of the problem mentioned is reduced. In case the problem mentioned still occurs, the significance of the error will be minimized as the probability increases (due to short distance between the relevant points from one and the adjacent sector) for the relevant point at the boundary of a sector and the adjacent relevant point at the boundary of the adjacent sector to contain the same or similar information on the visibility of the navigation marks (Figure 10).

The concept described has the potential to drastically reduce the probability of error, and in case an error still occurs, it is drastically reduced (due to a reduced distance between the relevant points). Naturally, the effectiveness of the concept mentioned will increase with an increase in the number of relevant points at the edges of a sector bordering other sectors, and with the relevant points located closer to the actual edges of the sector.

It is possible that the vessel is found exactly on the boundary between two sectors. That case is simply solved in the program so that it is explicitly determined for the boundary to which of the two sectors it belongs. Due to the concept mentioned from Figure 10 and due to short time intervals in which the web service and user (shipboard) application communicate, this case does not present a problem. 
It is also possible that the vessel is found at an equal distance between two or among more relevant points. The question then is to which of those relevant points the vessel will refer to considering that now there are two or even more closest relevant points. This case is also simply solved using the program in the way that one of these relevant points is randomly selected as the point of reference for the vessel. Like in the paragraph above, due to the concept mentioned from Figure 10 and short time intervals in which the web service and user (shipboard) application communicate, this case does not represent a problem.

A vessel cannot be found at the boundary of two UTM zones due to redundancy on the segments of axis $x$ ("easting") and axis y ("northing"), and also due to the fact that there is no GPS coordinate that simultaneously belongs to two UTM zones.

\section{ALGORITHM OF THE METHOD}

The method's operation principle is described in case the method operation logic is found entirely on the web service, and the user (shipboard) application serves only for transmitting the GPS coordinates of the vessel to the web service and for the display of information on the visibility of navigation marks that is sent by the web service back to the user (shipboard) application. In this case, the web service is also in charge of calculating the vessel's course.

An algorithm of the method is presented in steps $\mathbf{A}, \mathbf{B}$, C, D, E and F.

A) The user/shipboard application transmits two GPS coordinates to the web service. One coordinate marks the GPS position of the vessel at the time of transmission, and the other marks the previously stored GPS position of the vessel from the previous time. Further on, these are marked as "GPS current" and "GPS previous".

B) The web service searches by the multiple use of "ifelse" expressions to which sector the "GPS current " received by the user/shipboard application belongs.

C) Having detected the sector, the web service searches for the minimum distance between the "GPS current" and the relevant points from the database (or "cache" memory) that belong to this sector. In this search it uses the Euclidean distance for the two dimensions, but does not calculate roots, but only the squares of the Euclidean distances. In that way it finds the shortest distance, i.e. the closest relevant point.

D) Each relevant point contains a number of navigation marks and their GPS coordinates that are visible for that navigation mark, and now the web service retrieves from the database (or "cache" memory) the points of the visible navigation marks for that relevant point. The relevant point itself after this instant is of no further importance, and does not participate in the further steps in any way.

E) The web service now has at disposal the point "GPS current" and GPS points of the visible navigation marks for "GPS current" served by the closest relevant point. The web service calculates the Euclidean distance (with the calculated root), azimuth and bearing between the "GPS current" and each of the GPS points of the visible navigation marks.

The web service calculates the Euclidean distance (with the calculated root) d between "GPS current" and the first GPS point visible to the navigation mark. The web service determines in which quadrant there is the first GPS point of a visible navigation mark with regard to "GPS current". In other words, it is found out whether the navigation mark is located to the North-East (upper right quadrant of the 2D Cartesian coordinate system), NorthWest (upper left quadrant of the 2D Cartesian coordinate system), South-East (lower right quadrant) or SouthWest (lower left quadrant) with regard to "GPS current". Accordingly, the azimuth will be calculated in one of the four ways:

- 90 degrees - a (upper right quadrant),

- 90 degrees $+\alpha$ (lower right quadrant),

- 270 degrees + a (upper left quadrant) and

- 270 degrees - a (lower left quadrant).

$\alpha$ is the angle closed by the line that connects "GPS current" and GPS position of the navigation mark with the Equator line.

The expression $\sin$ is calculated:

$\sin \alpha=\frac{\Delta \text { northing }}{d}$

where $\Delta$ northing is the difference on "axis y" between "GPS current" and the GPS position of the navigation mark. From the expression mentioned above, it is then easy to calculate the angle $\alpha$. The web service now calculates the azimuth using one of the four above mentioned ways (four different quadrants) to calculate the azimuth.

The vessel's course is calculated using "GPS previous" in the same way the azimuth is calculated. In other words, to calculate the azimuth we use distance d, difference on "axis y" between "GPS current" and the GPS position of the navigation mark, and the above mentioned criterion for the calculation of the azimuth, while for the course the distance will be used between the position "GPS current" and "GPS previous", difference on "axis y" between "GPS current" and "GPS previous", and the same criterion based on quadrants that is used in azimuth calculation (Figure 11).

The web service now has at disposal the azimuth and the vessel's course, and the bearing is obtained by subtracting the course value from the azimuth value. If the difference of azimuth and course is negative, it is the matter of port bearing, and if it is positive, it is the matter of starboard bearing. The bearing cannot be greater than 180 degrees or smaller than -180 degrees. Thus, in case the bearing value obtained is larger than 180 degrees, it is necessary to subtract the obtained value from 360 degrees, so that now it represents a port bearing. In case 


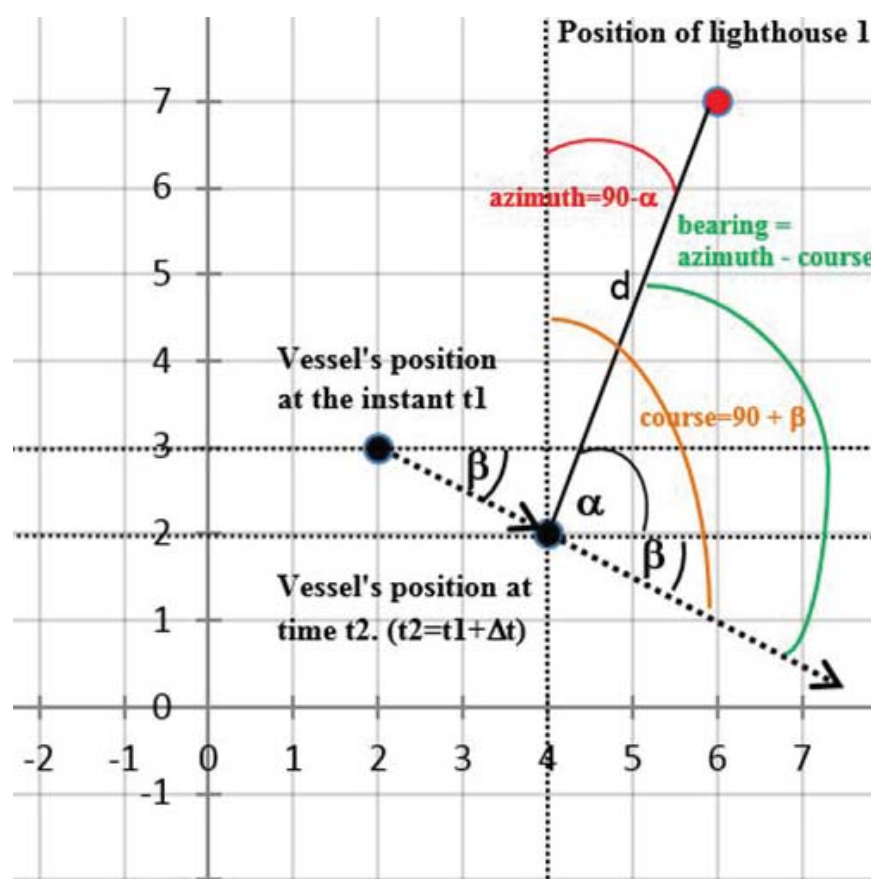

Figure 11: The concept of calculation of the vessel's course, distance, azimuth, and bearing between the vessel and navigation mark at an instant (Source: Authors)

the obtained value of the bearing is smaller than -180 degrees, then again from 360 degrees the obtained value is subtracted, and now it represents a starboard bearing.

The web service repeats this step E (except for the course calculation) for all the points of navigation marks that are visible to the current position of the vessel.

F) The web service sorts navigation marks (i.e. their names) together with the relative distances, azimuths, and bearings relative to the specific vessel in real-time communication (from the closest to the farthest navigation mark) and transmits to the user (shipboard) application which simply displays the information on the screen. The display can be performed as described in Figure 12.

\section{FEATURES OF THE METHOD}

There are several features of the method described:

- The method can be improved by extension with new relevant points to increase the method accuracy. For the same reason, the existing relevant points can be deleted and transferred if the need for this arises.

- In case new navigation marks occur, the method allows their easy import.

- The method can be extended to additional areas (addition of new sectors, relevant points).

- There is the possibility of introducing other ways of calculation of the minimum distances between the vessel's position and the positions of relevant points.

- Relevant points and navigation marks visible for each relevant point should be stored by an expert (seafarer, navigator).

- The criteria of number and distribution of the relevant points in an area can vary, and some of the criteria proposed to be considered when storing the relevant points are:

1. to increase the number of relevant points in places where skipper routes are more frequent,

2. to increase the number of relevant points in areas with a larger number of navigation marks than usual,

3. to increase the number of relevant points at the outer edges of the sector bordering other sectors, and to position the relevant points closer to the edges.

- The entire program logic of the method is provided on the independent web service, and the web service can be used by any application and any device.

\section{CONCLUSION}

During the high season period, the number of pleasure crafts and yachts increases. As a result, a higher number of accidents occurs. It is almost impossible to control such a large number of boats by Vessel Traffic Service $[17,18]$.

Solutions for fixing position and recognizing navigation marks with the help of web and internet is available in most coastal waters. This solution is relatively cheap and available, and of help to navigators, especially those who navigate for pleasure and in unfamiliar waters.

Fixing of position with the help of web and the internet is not professionally or SOLAS-approved. It has errors and it depends on the quality of internet communication and the quality of hardware. The use of such equipment

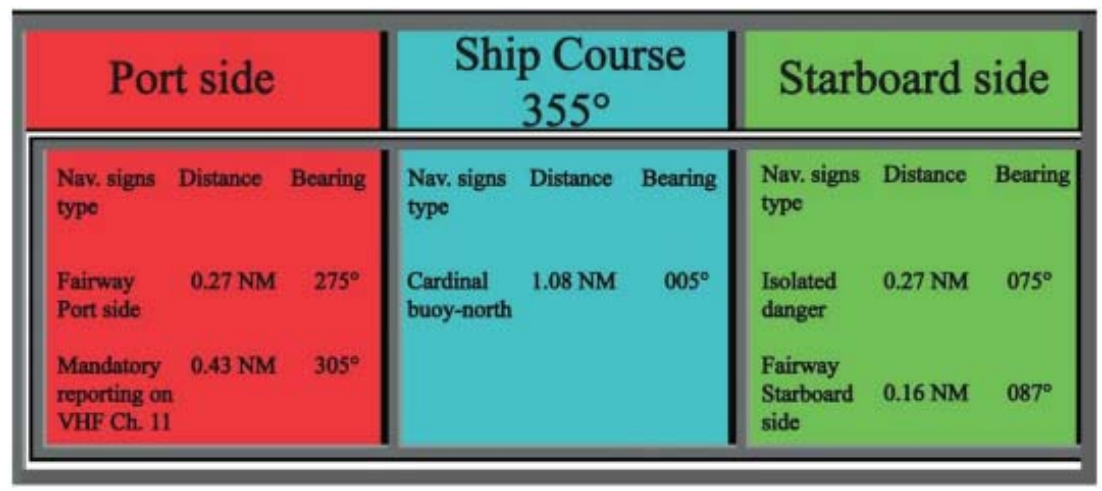

Figure 12: Example of the receiver displaying ship course $355^{\circ}$ [8] 
might contribute to safe navigation. Therefore, it should be taken into consideration as a way of reducing errors in the navigation of pleasure boats.

This is not a method of navigation, but might be a useful device. Using this new method and relative points could be helpful in recognizing navigational marks during navigation. Recognizing sea marks is helpful in the avoidance of errors in navigation and use not only GPS data in fixing the position of the boat.

This paper provides detailed descriptions of the novel method that identifies and presents/displays navigation marks to a navigator along with their distances, azimuths and bearings relative to the navigating vessel. An algorithm of the method is described in the context of the web solution, but it should be pointed out that the method itself can be implemented locally on a device with its own memory, without the presence of the Internet. The analysis of possible method issues is provided from the theoretical perspective. The implementation of the method in a practical technical/IT solution is planned for our future consideration.

\section{REFERENCES}

1. IALA. International Association of Marine Aids to Navigation and Lighthouse Authorities. (2017). IALA complementary lighthouse use manual. https://www. iala-aism.org/product-category/publications/manuals/ Accessed 12 February 2019

2. ILO. International Labour Organization. (2015). Seafarer Medical Fitness Standards - Anex I. http://www. lycee-maritime-ciboure.fr/files/Medical standards for_fitness_at_sea.pdf. Accessed 12 February 2019.

3. IHO. International Hydrographic Organization. (2010). Facts about Electronic Charts and Carriage Requirements. https://iho.int/iho_pubs/standard/S66/S-66_e1.0.0_EN.pdf. Accessed 12 February 2019.

4. IMO. International Maritime Organization. (1995). Resolution A.817(19). Performance standards for Electronic Chart Display and Information Systems (ECDIS). London: IMO. http://www.imo.org/en/ KnowledgeCentre/IndexofIMOResolutions/Assembly/Documents/A.817\%2819\%29.pdf. Accessed 12 February 2019.

5. SOLAS. International Convention for the Safety of Life at Sea, 1974. (2014). Chapter 5, Annex 3. Nautical Charts and Publications. Consolidated Edition 2014.

6. MAIB. Marine Accident Investigation Branch. (2009). Pride of Canter-bury Grounding Report. http://www. maib.gov.uk.

7. MAIB. Marine Accident Investigation Branch. (2012). CSL Thames Grounding Report. http://www.maib. gov.uk/ Accessed 12 February 2019.
8. Vidan, P. (2010). The model of increasing of safety on inland waterways. Doctoral thesis, Faculty of Traffic Sciences, Zagreb, 2010.

9. Movable Type Scripts. (2019). Calculate distance, bearing and more between Latitude/Longitude points. https://www.movable-type.co.uk/scripts/latlong.html. Accessed 12 February 2019.

10. Rosetta Code. (2016). Haversine formula. https://rosettacode.org/wiki/Haversine_formula. Accessed 12 February 2019.

11. Math Insight. (2019). Cartesian coordinates. https:// mathinsight.org/cartesian_coordinates. Accessed 12 February 2019.

12. NIMA. National Imagery and Mapping Agency. (2000). Department of Defense World Geodetic System 1984: Its Definition and Relationships with Local Geodetic Systems. http://earth-info.nga.mil/GandG/ publications/tr8350.2/wgs84fin.pdf. Accessed 12 February 2019.

13. Gratneer, C.E.(2017).HowtoFindEuclideanDistance. https://sciencing.com/euclidean-distance-7829754. html. Accessed 12 February 2019.

14. ArcGIS Resource Center. (2018). Coordinate systems, map projections, and geographic (datum) transformations. http://resources.esri.com/help/9.3/ ArcGISengine/dotnet/89b720a5-7339-44b0-8b58Of5bf2843393.htm. Accessed 12 December 2018.

15. Department of Geography. (2018). The UTM Grid and Transverse Mercator Projection. The Pennsylvania State University 2018. https://www.maptools. com/tutorials/grid zone details. Accessed 12 February 2019.

16. MapTools.(2019). MoredetailsaboutUTMGridZones. https://www.maptools.com/tutorials/grid_zone_details. Accessed 12 December 2018.

17. BSU. Federal Bureau of Maritime Casualty Investigation. (2009). LT Cortesia Grounding Report. http:// www.bsu-bund.de/EN. Accessed 12 December 2018.

18. BEA. French Marine Accident Investigation Office. (2010). Sischem Osprey Stranding Report.https://www. google.hr/url?sa=t\&rct=j\&q=\&esrc=s\&source=web\&c$\mathrm{d}=1$ \& ved =2ahUKEwil0Ivl33gAhWNjqQKHe2XAY4QFjAAegQIARAC\&url=https\%3A\%2F\%2Fwww.nautinst.org\%2Fdownload.cfm\%3Fdocid\%3DF9DA081F-6C1E-40F0 A71F0A89B10F426C\&usg=AOvVaw0d8BHGV2inL5CQBiDwvfG2. Accessed 12 December 2018.

Paper submitted: 16.10.2019.

Paper accepted: 31.10.2019.

This is an open access article distributed under the CC $B Y-N C-N D 4.0$ terms and conditions. 